\title{
Music therapy interventions in Parkinson's disease: the state-of-the-art
}

\author{
Alfredo Raglio* \\ Department of Public Health, Experimental and Forensic Medicine, University of Pavia, Pavia, Italy
}

Keywords: relational music therapy, neurological music therapy, rehabilitation, rhythm, motor symptoms, nonmotor symptoms

Parkinson's disease (PD) is a neurological disorder involving the progressive degeneration of the dopaminergic system, which gives rise to movement-related dysfunctions (such as bradykinesia, tremor, and rigidity) as well as other symptoms, mainly of cognitive and psychological nature. In the latter case, mood disorders prevails frequently causing anxiety and depression in all phases of the disease, sometimes even before the motor symptoms occur.

Aarsland and colleagues (1) report that $35 \%$ of the patients affected by PD present depression, whereas Richard (2) states that anxiety is to be found in $40 \%$ of the cases.

The literature shows that playing and listening to music may modulate emotions, behaviors, movements, communication, and cognitive factors, modifying the activity of the brain areas involved in the perception and regulation of these aspects $(3,4)$.

Music can produce substantial effects on movement-related symptoms as well as psychological ones in PD treatment. Concerning the first aspect, rhythm has a crucial role in rehabilitation, enhancing connections between the motor and auditory systems (5).

Literature showed how a rhythmic auditory cues-based training can produce a compensation of the cerebello-thalamo-cortical network leading to beneficial effects, for example, improving not only speed and step length but also perceptual and motor timing abilities $(6,7)$.

Areas involving rhythm perception are closely related to those that regulate movement (such as the premotor cortex, supplementary motor area, cerebellum, and basal ganglia - especially putamen) (8-18). A study conducted with fMRI (19) shows that whereas a regular pulse (in contrast to an irregular one) generally activates basal ganglia in a significant way, this is not the case in PD. Other studies $(7,20)$ support the idea that external cues (in particular rhythmical cues) can modulate the activity within the impaired timing system. This may mean that a regular rhythmic pulse stimulates the putamen activity, facilitating movement and providing an input for sequential movements and impaired automatized processes. Moreover, this could compensate for the lack of dopaminergic stimulation.

Rhythm can be also perceived visually and through the tactile sense, but the reaction time of the human auditory system is shorter by $20-50 \mathrm{~ms}$, when compared to visual and tactile stimuli; moreover, it has a stronger capacity of perceiving rhythm periodicity and structure (6). Therefore, rhythm influences the kinetic system (through synchronization and adjustment of muscles to auditory stimuli), facilitates movement synchronization, coordination, and regularization, and may even produce an internal rhythm that persists in the absence of stimuli (21-23).

Many studies report that musical rhythm in PD treatment can improve gait (speed, frequency, and step length), limbs coordination, postural control, and balance $(7,18,24-36)$. In view of the above, Neurologic Music Therapy (NMT) - especially Rhythmic Auditory Stimulation, one of its techniques - characterizes this approach to the disease: NMT aims at enhancing sensory, cognitive, and motor functions (as in PD treatment, in which specific rhythmic techniques can strengthen and improve the rehabilitative process). 
Studies pertaining to non-motor symptoms (e.g., psychological aspects, such as anxiety or depression) are fewer in number, and therefore results are less certain $(24,36-41)$. From this point of view, music therapy approaches focused on the relationship between music therapist and patient serve as interventions that can produce substantial psychological improvements, creating moments of empathetic relationship and emotional connection. Sound is indeed an important means of communication, especially in a non-verbal context, promoting emotional expression and regulation. In PD treatment, the music therapeutic approach described above, based on free improvisation, is most assuredly less used, or maybe less documented. Sound/music improvisation can be considered as the main technique in the active music therapy approaches (42). During the improvisation, music therapist and patient interact freely using musical instruments, generally without musical rules or themes.

Moreover, making, and listening to, music can be considered as strong stimuli from the emotional point of view, playing an important role in the activation of the limbic system and neuro-chemical circuits (i.e., of the reward system) $(43,44)$.

A distinction between the relational and rehabilitative approach is extremely important, not only to determine the type of intervention but also to define the specific identity of the music therapy approach. In the former, the music therapist aims at building a relationship with the patient by means of interaction with melodic and rhythmical instruments and singing (improvisation). This facilitates the expression and modulation of patients' emotions and promotes communication and empathetic relationships. In the latter, the music therapist proposes music-based exercises (in particular using rhythmical patterns) to improve motor, cognitive, and sensory functions, generally impaired by neurological damage. Although the two methods are often mutually influenced, there is a substantial difference in terms of goals, intervention models, techniques, and music therapy training. From this perspective, I believe that research should take these aspects into account, in order to focus on goals more accurately, while maintaining the possibility of overlapping the two approaches - making them complementary rather than antithetical. Often literature does not consider the distinction among these different music therapy approaches, defining every experience as "music therapy." In contrast to this, I believe that the achievement of an adequate definition regarding different types of intervention is necessary; it should also highlight different theoretical and methodological bases and their different areas of application. To this end, a recent article by Raglio and Oasi (45) reports the main characteristics of music-based interventions, deriving from an in-depth analysis of their related contents and of literature.

Table 1 shows how clinical studies characterized by strong scientific implications (Randomized Controlled Trials and Clinical Controlled Trials) and based on rehabilitative treatments (not on evaluating tasks in the presence of a musical stimulus) are lacking in literature. The table summarizes the main effects produced on motor and non-motor symptoms and distinguishes the various interventions in terms of different musical contents and techniques.

In PD treatment based on NMT, it is important to understand what specifically acts on the disease: it is unclear, for example, whether the beat produces an effect by itself in neuromotor rehabilitation or, in contrast, the effects are produced by music

TABLE 1 | Randomized Controlled Trials and Controlled Clinical Trials from PubMed database regarding Parkinson's disease, including clinical studies (based on rehabilitative treatments) in English.

\begin{tabular}{|c|c|c|c|c|c|}
\hline Reference & Motor outcomes & Non-motor outcomes & $\begin{array}{l}\text { Subjects } \\
n \text { experimental } \\
\text { group }+n \\
\text { control group }\end{array}$ & Interventions/duration & Follow-up \\
\hline (7) & $\begin{array}{l}\text { Improving in perceptual and } \\
\text { motor timing }\end{array}$ & Not evaluated & $15+20$ & $\begin{array}{l}\text { Rhythmic auditory cueing (beat + superimposed } \\
\text { familiar songs)/4 weeks ( } 30 \text { min/session, } \\
3 \text { sessions/week) }\end{array}$ & Yes \\
\hline (24) & $\begin{array}{l}\text { Improving over time in motor } \\
\text { function, cognitive function } \\
\text { (verbal memory, language, and } \\
\text { executive function and attention) }\end{array}$ & $\begin{array}{l}\text { Slight improvement over time } \\
\text { quality of life }\end{array}$ & $12+6$ & $\begin{array}{l}\text { Ronnie Gardiner rhythm and music (music } \\
\text { method uses music, rhythm, movements and } \\
\text { speech)/6 weeks ( } 1 \text { h/session, } 2 \text { sessions/week) }\end{array}$ & None \\
\hline (25) & $\begin{array}{l}\text { Improving in functional gait, } \\
\text { balance, and freezing }\end{array}$ & Not evaluated & $8+8$ & $\begin{array}{l}\text { Rhythmic auditory stimulation (RAS)/6 weeks } \\
\text { (45-60 min/session, 3 sessions/week) }\end{array}$ & Yes \\
\hline$(40)$ & $\begin{array}{l}\text { Slight improvement over time in } \\
\text { tremor }\end{array}$ & $\begin{array}{l}\text { Improving over time in mood } \\
\text { and anxiety; modest } \\
\text { improvement on quality of life }\end{array}$ & $18+18$ & $\begin{array}{l}\text { Music relaxation/4 weeks ( } 45 \mathrm{~min} / \text { session, } \\
2 \text { sessions/week) }\end{array}$ & Yes \\
\hline$(41)$ & $\begin{array}{l}\text { Significant improvement in } \\
\text { bradykinesia }\end{array}$ & $\begin{array}{l}\text { Improving over time in } \\
\text { emotional functions, activities } \\
\text { of daily living and quality of life }\end{array}$ & $16+16$ & $\begin{array}{l}\text { Music therapy sessions (choral singing, voice } \\
\text { exercise, rhythmic and free body movements, and } \\
\text { improvisational music therapy techniques)/ } \\
3 \text { months ( } 2 \text { h/session, weekly sessions) }\end{array}$ & Yes \\
\hline (34) & $\begin{array}{l}\text { Improving in gait parameters } \\
\text { (velocity, stride length and step } \\
\text { cadence) }\end{array}$ & Not evaluated & $15+11$ & $\begin{array}{l}\text { Rhythmic auditory stimulation (RAS) } \\
\text { (beat + superimposed music)/3 weeks } \\
\text { (30 min/session, daily) }\end{array}$ & None \\
\hline
\end{tabular}

Search criteria: ("music" OR "music therapy" OR "rhythmic auditory stimulation") AND "Parkinson." 
(that we can consider the sum and combination of rhythm, melody, harmony, and other parameters) with a marked pulse (6). In fact, if music is excluded from interventions as described in many studies, we have to ask ourselves what then is the role of music therapists; furthermore, should such rhythm-based interventions be considered as part of music therapy programs, or rather simply as rehabilitative methods supported by rhythm, given that music does not assume a crucial role.

Additional weak points of NMT studies are given by the small sample size, the short duration of treatments and by the frequent lack of medium and long-term assessment (follow-up).

Another consideration is connected with the possibility to prove whether an effect produced during rehabilitation can persist also in the absence of stimuli. In this sense, the aforementioned studies conducted by Benjamin (21), Jackendoff (22), and Palmer and Krumhansl (23) offer various starting points that should be discussed in-depth, concerning the potential of the retrieval of imagined rhythm/music components, even after the stimuli have ceased or are absent (46).

A limited number of studies take the psychological outcome into account; these studies propose techniques based on a relational approach, which aims to develop an empathetic communication with the patient through the medium of sound. In music therapy, the active relational approach is based mainly on improvisation (42), and, from a practical standpoint, emphasizes the chances of enhancing and synchronizing the patient's movements. When reviewing videotapes of the therapeutic sessions based on this approach, it becomes clear that there are significant changes in regularity and fluency of movement of the upper limbs that also

\section{References}

1. Aarsland D, Påhlhagen S, Ballard CG, Ehrt U, Svenningsson P. Depression in Parkinson disease: epidemiology, mechanisms and management. Nat Rev Neurol (2011) 8(1):35-47. doi:10.1038/nrneurol.2011.189

2. Richard IH. Anxiety disorders in Parkinson's disease. Adv Neurol (2005) 96:42-55.

3. Hillecke T, Nickel A, Bolay HV. Scientific perspectives on music therapy. Ann N Y Acad Sci (2005) 1060:271-82. doi:10.1196/annals.1360.020

4. Koelsch S. A neuroscientific perspective on music therapy. Ann N Y Acad Sci (2009) 1169:374-84. doi:10.1111/j.1749-6632.2009.04592.x

5. Thaut MH. Rhythm, Music and the Brain: Scientific Foundations and Clinical Applications. New York, NY: Taylor \& Francis Group (2005).

6. Nombela C, Hughes LE, Owen AM, Grahn JA. Into the groove: can rhythm influence Parkinson's disease? Neurosci Biobehav Rev (2013) 37:2564-70. doi: 10.1016/j.neubiorev.2013.08.003

7. Benoit CE, Dalla Bella S, Farrugia N, Obrig H, Mainka S, Kotz SA. Musically cued gait-training improves both perceptual and motor timing in Parkinson's disease. Front Hum Neurosci (2014) 8:494. doi:10.3389/fnhum.2014.00494

8. Bijsterbosch JD, Lee KH, Hunter MD, Tsoi DT, Lankappa S, Wilkinson ID, et al. The role of the cerebellum in sub- and supraliminal error correction during sensorimotor synchronization: evidence from fMRI and TMS. J Cogn Neurosci (2011) 23(5):1100-12. doi:10.1162/jocn.2010.21506

9. Thaut MH, Stephan KM, Wunderlich G, Schicks W, Tellmann L, Her$\operatorname{zog} \mathrm{H}$, et al. Distinct cortico-cerebellar activations in rhythmic auditory motor synchronization. Cortex (2009) 45(1):44-53. doi:10.1016/j.cortex.2007. 09.009

10. Grahn JA, Rowe JB. Feeling the beat: premotor and striatal interactions in musicians and nonmusicians during beat perception. J Neurosci (2009) 29(23):7540-8. doi:10.1523/JNEUROSCI.2018-08.2009

11. Bengtsson SL, Ullén F, Ehrsson HH, Hashimoto T, Kito T, Naito E, et al. Listening to rhythms activates motor and premotor cortices. Cortex (2009) 45(1):62-71. doi:10.1016/j.cortex.2008.07.002 positively influence the patient's sound/music production. The music therapy relational approach in PD treatment is, therefore, less usual but equally as important; it is, in fact, less performancecentered and takes the patient's personal expression into account, thus allowing a dynamic calibration of the stimulus, through a process of continuous regulation, combining and integrating the physical and psychological components. Considerations emerging from the relational music therapeutic experience in PD treatment emphasize the rhythmic component of improvisation, leading the patient into synchronization, at the same time maintaining freedom of expression and a non-prescriptive approach based on the free sonorous-music improvisation. This facilitates the integration of psychological aspects (the empathetic relationship, the regulation, and modulation of emotional expressions) with motor functions (speed and fluency of movement, in particular of the upper limbs).

Therefore, interventions that involve music can offer important starting points in PD rehabilitation, effectively acting on motor, as well as non-motor symptoms. In this sense, research should increase the number of studies based on strong methodological criteria, also including a clear description of the intervention whether it be relational or rehabilitating - a consistent and numerically significant sample, in addition to more sensitive tools to evaluate motor and psychological outcomes. In conclusion, a stronger research methodology and a clearer definition of the exact medium or parameter in music related to specific output of rehabilitation are needed. This will allow the development of adequate, and increasingly specific and effective music therapy approaches.

12. Chen JL, Penhune VB, Zatorre RJ. Listening to musical rhythms recruits motor regions of the brain. Cereb Cortex (2008) 18(12):2844-54. doi:10.1093/cercor/ bhn042

13. Grahn JA, Brett M. Rhythm and beat perception in motor areas of the brain. J Cogn Neurosci (2007) 19(5):893-906. doi:10.1162/jocn.2007.19.5.893

14. Lewis PA, Miall RC. Distinct systems for automatic and cognitively controlled time measurement: evidence from neuroimaging. Curr Opin Neurobiol (2003) 13(2):250-5. doi:10.1016/S0959-4388(03)00036-9

15. Mayville JM, Fuchs A, Ding M, Cheyne D, Deecke L, Kelso JA. Eventrelated changes in neuromagnetic activity associated with syncopation and synchronization timing tasks. Hum Brain Mapp (2001) 14(2):65-80. doi:10. 1002/hbm.1042

16. Schubotz RI, von Cramon DY. Interval and ordinal properties of sequences are associated with distinct premotor areas. Cereb Cortex (2001) 11(3):210-22. doi:10.1093/cercor/11.3.210

17. Ullén F, Bengtsson SL. Independent processing of the temporal and ordinal structure of movement sequences. J Neurophysiol (2003) 90(6):3725-35. doi:10. 1152/jn.00458.2003

18. McIntosh GC, Brown SH, Rice RR, Thaut MH. Rhythmic auditory-motor facilitation of gait patterns in patients with Parkinson's disease. J Neurol Neurosurg Psychiatry (1997) 62(1):22-6. doi:10.1136/jnnp.62.1.22

19. Grahn JA. The role of the basal ganglia in beat perception: neuroimaging and neuropsychological investigations. Ann N Y Acad Sci (2009) 1169:35-45. doi:10.1111/j.1749-6632.2009.04553.x

20. Jahanshahi M, Jenkins IH, Brown RG, Marsden CD, Passingham RE, Brooks DJ. Self-initiated versus externally triggered movements. I. An investigation using measurement of regional cerebral blood flow with PET and movement-related potentials in normal and Parkinson's disease subjects. Brain (1995) 118:913-33. doi:10.1093/brain/118.4.913

21. Benjamin WE. A theory of musical meter. Music Percept (1984) 1:355-413. doi:10.2307/40285269

22. Jackendoff RS. A Generative Theory of Tonal Music. Cambridge, IN: MIT Press (1983). 
23. Palmer C, Krumhansl CL. Mental representations for musical meter. J Exp Psychol Hum Percept Perform (1990) 16:728-41. doi:10.1037/0096-1523.16.4.728

24. Pohl P, Dizdar N, Hallert E. The Ronnie Gardiner rhythm and music method - a feasibility study in Parkinson's disease. Disabil Rehabil (2013) 35(26):2197-204. doi:10.3109/09638288.2013.774060

25. Kadivar Z, Corcos DM, Foto J, Hondzinski JM. Effect of step training and rhythmic auditory stimulation on functional performance in Parkinson patients. Neurorehabil Neural Repair (2011) 25(7):626-35. doi:10.1177/ 1545968311401627

26. Thaut MH, Abiru M. Rhythmic auditory stimulation in rehabilitation of movement disorders: a review of current research. Music Percept (2010) 27:263-9. doi:10.1525/MP.2010.27.4.263

27. Rochester L, Burn DJ, Woods G, Godwin J, Nieuwboer A. Does auditory rhythmical cueing improve gait in people with Parkinson's disease and cognitive impairment? A feasibility study. Mov Disord (2009) 24(6):839-45. doi:10.1002/ mds. 22400

28. Arias P, Cudeiro J. Effects of rhythmic sensory stimulation (auditory, visual) on gait in Parkinson's disease patients. Exp Brain Res (2008) 186(4):589-601. doi:10.1007/s00221-007-1263-y

29. Satoh M, Kuzuhara S. Training in mental singing while walking improves gait disturbance in Parkinson's disease patients. Eur Neurol (2008) 60(5):237-43. doi:10.1159/000151699

30. del Olmo MF, Cudeiro J. Temporal variability of gait in Parkinson disease: effects of a rehabilitation programme based on rhythmic sound cues. Parkinsonism Relat Disord (2005) 11(1):25-33. doi:10.1016/j.parkreldis.2004.09.002

31. Lim I, van Wegen E, de Goede C, Deutekom M, Nieuwboer A, Willems A, et al. Effects of external rhythmical cueing on gait in patients with Parkinson's disease: a systematic review. Clin Rehabil (2005) 19(7):695-713. doi:10.1191/ 0269215505 cr906oa

32. Bernatzky G, Bernatzky P, Hesse HP, Staffen W, Ladurner G. Stimulating music increases motor coordination in patients afflicted with Morbus Parkinson. Neurosci Lett (2004) 361(1-3):4-8. doi:10.1016/j.neulet.2003.12.022

33. Thaut MH, McIntosh KW, McIntosh GC, Hoemberg V. Auditory rhythmicity enhances movement and speech motor control in patients with Parkinson's disease. Funct Neurol (2001) 16(2):163-72.

34. Thaut MH, McIntosh GC, Rice RR, Miller RA, Rathbun J, Brault JM. Rhythmic auditory stimulation in gait training for Parkinson's disease patients. Mov Disord (1996) 11(2):193-200. doi:10.1002/mds.870110213

35. Hurt CP, Rice RR, McIntosh GC, Thaut MH. Rhythmic auditory stimulation in gait training for patients with traumatic brain injury. J Music Ther (1998) 35(4):228-41. doi:10.1093/jmt/35.4.228

36. Miller RA, Thaut MH, McIntosh GC, Rice RR. Components of EMG symmetry and variability in parkinsonian and healthy elderly gait. Electroencephalogr Clin Neurophysiol (1996) 101(1):1-7. doi:10.1016/0013-4694(95)00209-X
37. De Dreu MJ, van der Wilk AS, Poppe E, Kwakkel G, van Wegen EE. Rehabilitation, exercise therapy and music in patients with Parkinson's disease: a meta-analysis of the effects of music-based movement therapy on walking ability, balance and quality of life. Parkinsonism Relat Disord (2012) 18:S114-9. doi:10.1016/S13538020(11)700360

38. Elefant C, Baker FA, Lotan M, Lagesen SK, Skeie GO. The effect of group music therapy on mood, speech, and singing in individuals with Parkinson's disease - a feasibility study. J Music Ther (2012) 49(3):278-302. doi:10.1093/ jmt/49.3.278

39. Hayashi A, Nagaoka M, Mizunu Y. Music therapy in Parkinson's disease: improvement of parkinsonian gait and depression with rhythmic auditory stimulation. Parkinsonism Relat Disord (2006) 12(Suppl 2):S76. doi:10.1016/j. parkreldis.2006.05.026

40. Craig LH, Svircev A, Haber M, Juncos JL. Controlled pilot study of the effects of neuromuscular therapy in patients with Parkinson's disease. Mov Disord (2006) 21(12):2127-33. doi:10.1002/mds.21132

41. Pacchetti C, Mancini F, Aglieri R, Fundarò C, Martignoni E, Nappi G. Active music therapy in Parkinson's disease: an integrative method for motor and emotional rehabilitation. Psychosom Med (2000) 62:386-93. doi:10.1097/00006842200005000-00012

42. Gold C, Solli HP, Krüger V, Lie SA. Dose-response relationship in music therapy for people with serious mental disorders: systematic review and meta-analysis. Clin Psychol Rev (2009) 29(3):193-207. doi:10.1016/j.cpr.2009. 01.001

43. Koelsch S. Brain correlates of music-evoked emotions. Nat Rev Neurosci (2014) 15(3):170-80. doi:10.1038/nrn3666

44. Chanda ML, Levitin DJ. The neurochemistry of music. Trends Cogn Sci (2013) 17(4):179-93. doi:10.1016/j.tics.2013.02.007

45. Raglio A, Oasi O. Music and health: what interventions for what results? Front Psychol (2015) 6:230. doi:10.3389/fpsyg.2015.00230

46. Schaefer RS, Morcom AM, Roberts N, Overy K. Moving to music: effects of heard and imagined musical cues on movement-related brain activity. Front Hum Neurosci (2014) 8:774. doi:10.3389/fnhum.2014.00774

Conflict of Interest Statement: The author declares that the research was conducted in the absence of any commercial or financial relationships that could be construed as a potential conflict of interest.

Copyright (c) 2015 Raglio. This is an open-access article distributed under the terms of the Creative Commons Attribution License (CC BY). The use, distribution or reproduction in other forums is permitted, provided the original author(s) or licensor are credited and that the original publication in this journal is cited, in accordance with accepted academic practice. No use, distribution or reproduction is permitted which does not comply with these terms. 